\title{
Analysis of Undirected Formation Shape Control with Directional Mismatch
}

\author{
Ziyang Meng, Brian D. O. Anderson and Sandra Hirche
}

\begin{abstract}
A typical formation shape control problem involves point agents sensing relative positions, i.e. directions and distances, of their neighbors and then moving so that these relative positions achieve some prespecified values. Such a procedure requiring as it does sensing of directions implicitly presupposes that all agents have a shared understanding of the direction of north. On the other hand, there may be biases in sensors, variations in the earths magnetic field interfering with compass-based sensing, or drift in inertial sensors, with the result that directions are inconsistently measured or measured with error. This paper considers the consequences of this error, considering first the two agent case in a two-dimensional plane. We show that the agents converge to a fixed, but distorted formation exponentially fast. In contrast to the matched case, the formation is not asymptotically stationary, but rather instead translates with a certain constant velocity. The distance error and the angular error between the actual final formation and the desired formation are explicitly given, as well as the steady state velocity of the formation. Based on the results, estimation algorithm is given to obtain the mismatch angle, which allows a compensation algorithm to be proposed such that the desired formation is achieved with zero steady-state velocity for the formation as a whole. The case of the threedimensional ambient space is then considered and similar phenomena are observed. Simulations are also provided to validate the theoretical results.
\end{abstract}

\section{INTRODUCTION}

Formation shape control problems are considered an important issue in the study of multi-agent systems and shape control has broad applications [1], [2]. There are different variations for the formation shape control problem. For example, problems with or without a leader were considered in [3], [4]; problems with undirected or directed communication topology were studied in [5], [6] and problems with velocity consensus and moving final formation were investigated in [7], [8]. Another major distinctions rest with problems where there are both a prescribed shape and a prescribed orientation, and problems seeking simply to achieve targeting on a prescribed shape. A linear consensus-based algorithm can be used to solve a formation shape control problem with both a prescribed shape and a prescribed orientation [9], [10] while the gradient-based approach (which involves

Z. Meng is with Department of Precision Instrument, Tsinghua University, Beijing 100084, China. B. D. O. Anderson is with The Australian National University and National ICT Australia, Canberra ACT 2600 Australia. S. Hirche is with Institute for InformationOriented Control, Technische Universität München, D-80290 Munich, Germany. Email: \{ziyang.meng, hirche\}@tum.de, \{brian.anderson\}eanu.edu.au. Corresponding author: Z. Meng. NICTA is supported by the Australian Government under the ICT Centre of Excellence Program. The work was also supported by the Australian Research Council under grants DP110100538 and DP130103610, and the Alexander von Humboldt Foundation of Germany. nonlinear control) can be used for shape control without orientation objective [11], [12]. In this paper, the consensusbased approach is considered. An indispensable assumption for consensus-based approach is that all agents need the knowledge of the direction of the common/global north. Although common knowledge of north can be secured by assuming that all agents have access to accurate GPS data, this can be an overly-ambitious assumption indoors or in a military environment. Therefore, a more practical approach is to require all agents to be equipped with some form of compass.

It is however evident that it will often be unrealistic to claim that all agents have common error-free knowledge of where north is: biases can exist in instruments; drift can occur in inertial navigation systems; spatial variation can occur in the earth's magnetic field, and so on. This paper explores the consequence of postulating the existence of errors in the direction of north, i.e. agents have differing views of where north is. To illustrate the key concepts, we start from the matched formation shape control for two agents in a twodimensional plane,

$$
\begin{aligned}
& \dot{\mathbf{A}}_{1}=\left(\mathbf{A}_{2}-\mathbf{A}_{1}\right)-\mathbf{D}, \\
& \dot{\mathbf{A}}_{2}=\left(\mathbf{A}_{1}-\mathbf{A}_{2}\right)+\mathbf{D},
\end{aligned}
$$

where $\mathbf{A}_{1}=\left[x_{1}, y_{1}\right]^{\mathrm{T}} \in \mathbb{R}^{2}$ and $\mathbf{A}_{2}=\left[x_{2}, y_{2}\right]^{\mathrm{T}} \in \mathbb{R}^{2}$ are the positions of agents 1 and $2, \mathbf{V}_{1}=\dot{\mathbf{A}}_{1}$ and $\mathbf{V}_{2}=$ $\dot{\mathbf{A}}_{2}$ represent the velocities of agents 1 and 2 , and $\mathbf{D}=$ $\left[d_{x}, d_{y}\right]^{\mathrm{T}} \in \mathbb{R}^{2}$ is a given desired relative position and known for each agent. The objective is to drive agents 1 and 2 to form a steady formation in the plane such that $\mathbf{A}_{2}=\mathbf{A}_{1}+\mathbf{D}$. Note that the position of the centroid is unspecified, while the orientation and shape of the formation are specified. It is straightforward from (1) to show that $\lim _{t \rightarrow \infty}\left(\mathbf{A}_{2}(t)-\right.$ $\left.\mathbf{A}_{1}(t)\right)=\mathbf{D}, \lim _{t \rightarrow \infty} \dot{\mathbf{A}}_{1}(t)=0$, and $\lim _{t \rightarrow \infty} \dot{\mathbf{A}}_{2}(t)=0$ exponentially fast. Therefore, agents converge to the desired formation and the velocities converge to zero exponentially fast.

The above algorithm assumes that $\mathbf{A}_{2}-\mathbf{A}_{1}$ for agent 1 and $\mathbf{A}_{1}-\mathbf{A}_{2}$ for agent 2 are identical (up to the sign). This means that a global coordinate system is shared for the two agents. However, this assumption is unlikely to be satisfied in real systems. As already indicated, we will focus on the directional (as opposed to range) error, i.e., error maybe arising from a compass mismatch.

Without any loss of generality, we suppose that the global coordinates coincide with the coordinate basis of agent 1 . We next derive the equation of motion of agent 2 in global 


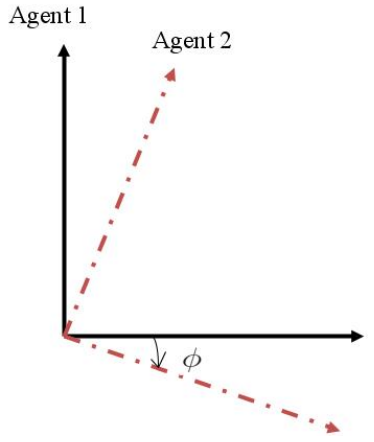

Fig. 1. Coordinates of agents 1 and 2 .

coordinates. Suppose $\mathbf{A}_{i}$ denotes the position of agent $i$, $i=1,2$ in global coordinates and ${ }^{2} \mathbf{A}_{i}$ denotes its position in agent 2's coordinates. Suppose agent 2's view of north is that it is $\phi$ radians in a counterclockwise direction from agent 1's view, where $\phi \in(-\pi, \pi]$ and Figure 1 gives an illustration. Therefore, a line vector in global coordinates ${ }^{1} v=[x, y]^{\mathrm{T}}$ can be described in agent 2's coordinate basis as ${ }^{2} v=R(\phi)^{1} v$, where $R(\phi)=\left[\begin{array}{cc}\cos \phi & -\sin \phi \\ \sin \phi & \cos \phi\end{array}\right]$ is the rotation matrix.

Then, in each agent's own coordinate basis, the actual kinematics of each agent with mismatched directions are given by

$$
\begin{gathered}
{ }^{1} \dot{\mathbf{A}}_{1}=\mathbf{A}_{2}-\mathbf{A}_{1}-\mathbf{D} \\
{ }^{2} \dot{\mathbf{A}}_{2}=R(\phi)\left(\mathbf{A}_{1}-\mathbf{A}_{2}\right)+\mathbf{D}
\end{gathered}
$$

where $\mathbf{A}_{1}-\mathbf{A}_{2}$ is expressed in global coordinates, ${ }^{1} \dot{\mathbf{A}}_{1}$ and ${ }^{2} \dot{\mathbf{A}}_{2}$ are the velocity vectors of agents 1 and 2 expressed in each agent's own coordinate basis.

The authors of [13] considered a similar problem with an orientation mismatch of local reference frames of the agents for the formation shape control problem. Under the assumption that the orientation of each agent can be exchanged, both orientation alignment control algorithm and formation shape control algorithm were proposed. Distance errors have been considered in the context of formation shape control without orientation in [14], [15]. It was shown in [14] that if the agents have different understandings on either the desired distance between them, or of the actual distance between them, the resulting steady state formation will be of fixed shape but distorted relative to the desired shape (the amount of distortion depending on the mismatch). Instead of being stationary, the resulting formation shape will converge to a circular closed orbit in two-dimensional plane. The radius may be large, but the angular velocity is proportional to the mismatch. The extension to the case of a three-dimensional tetrahedron formation shape control problem was considered in [15] and it was shown that the motion behavior is typically a helix and attributable to mismatch in desired or measured distances for a pair of agents.

In this paper, we first consider the directional mismatch problem modelled by (2) in a two-dimensional plane and then study the case of a three-dimensional ambient space. In particular, we show that the agents converge to a fixed, but (relative to the desired one) distorted formation exponentially fast for both cases. The shape error between the actual final formation and the desired formation is explicitly given and is roughly proportional to the angular mismatch in the coordinate axis directions of the two agents. An estimation algorithm for the mismatched angle is also proposed. Based on the design of the estimator, which is incorporated in a more complicated control, a mismatch compensation algorithm is proposed such that the desired formation is achieved and in steady state, it is stationary. We finally include discussions on the case of a three-dimensional ambient space.

Notation: $\|x\|$ denote the 2-norm of a vector $x \in \mathbb{R}^{d}$. Let $f$ and $g$ be two functions defined on some subset of the real numbers. One writes $f(x)=O(g(x))$ as $x \rightarrow 0$ if and only if there exists a positive real numbers $M$ and $\delta$ such that $|f(x)| \leq M|g(x)|$ for $|x|<\delta$.

\section{TWO-DIMENSIONAL PLANE CASE}

Let us go back to (2) and assume that $\phi$ is constant. By noting the fact that ${ }^{1} \dot{\mathbf{A}}_{2}=R(-\phi)^{2} \dot{\mathbf{A}}_{2}$, it is not hard to show that (2) can be written as

$$
\begin{gathered}
\dot{\mathbf{A}}_{1}=\mathbf{A}_{2}-\mathbf{A}_{1}-\mathbf{D}, \\
\dot{\mathbf{A}}_{2}=\mathbf{A}_{1}-\mathbf{A}_{2}+R(-\phi) \mathbf{D},
\end{gathered}
$$

where $\mathbf{A}_{1}, \mathbf{A}_{2}, \dot{\mathbf{A}}_{1}$, and $\dot{\mathbf{A}}_{2}$ are all expressed in global coordinates. We next focus on (3) and study both the intraformation motion and whole-formation motion of the closedloop system.

\section{A. Behaviors of the formation}

We first establish the following result on how the formation of two agents evolves when there exist directional mismatches.

Theorem 1: Consider the mismatched formation shape control algorithm (2). Suppose $\phi \neq 0$ and constant. It follows that

[Intra-formation motion]

(I) The agents converge to a fixed formation exponentially fast. In particular, $\lim _{t \rightarrow \infty}\left(\mathbf{A}_{2}(t)-\mathbf{A}_{1}(t)\right)=$ $\cos \frac{\phi}{2} R(-\phi / 2) \mathbf{D}$.

(II) The relative velocities of the agents converge to zero exponentially fast, i.e., $\lim _{t \rightarrow \infty}\left(\mathbf{V}_{2}(t)-\mathbf{V}_{1}(t)\right)=0$.

(III) If $\mathbf{D} \neq 0$, the final formation is distorted from the desired one. The distance error and the angular error between the actual final relative position and the desired one are $O\left(\phi^{2}\right)$, as $\phi \rightarrow 0$ and $\frac{|\phi|}{2}$, respectively. If $\mathbf{D}=0$, then rendezvous is still achieved, i.e., $\lim _{t \rightarrow \infty}\left(\mathbf{A}_{2}(t)-\mathbf{A}_{1}(t)\right)=0$.

[Whole-formation motion]

(IV) If $\mathbf{D} \neq 0$, the absolute velocities of each agent converge to the same nonzero constant exponentially fast. In particular, $\lim _{t \rightarrow \infty} \mathbf{V}_{1}(t)=\lim _{t \rightarrow \infty} \mathbf{V}_{2}(t)=$ $\sin \frac{\phi}{2}\left[\begin{array}{cc}\sin \frac{\phi}{2} & \cos \frac{\phi}{2} \\ -\cos \frac{\phi}{2} & \sin \frac{\phi}{2}\end{array}\right] \mathbf{D}$ and $\lim _{t \rightarrow \infty}\left\|\mathbf{V}_{1}(t)\right\|=$ 
$\lim _{t \rightarrow \infty}\left\|\mathbf{V}_{2}(t)\right\|=O(|\phi|)$, as $\phi \rightarrow 0$. If $\mathbf{D}=0$, the absolute velocities of both agents converge to zero exponentially fast.

(V) If $\mathbf{D} \neq 0$, the agent positions $\mathbf{A}_{1}(t)$ and $\mathbf{A}_{2}(t)$ are neither convergent nor bounded. If $\mathbf{D}=0$, $\lim _{t \rightarrow \infty} \mathbf{A}_{1}(t)=\lim _{t \rightarrow \infty} \mathbf{A}_{2}(t)=\frac{\mathbf{A}_{1}(0)+\mathbf{A}_{2}(0)}{2}$, where $\mathbf{A}_{1}(0)$ and $\mathbf{A}_{2}(0)$ are the initial states of agents 1 and 2.

Proof:

(I) Define $\mathbf{Q}=\mathbf{A}_{1}-\mathbf{A}_{2}+\frac{1}{2}(I+R(-\phi)) \mathbf{D}$. It follows from (3) that

$$
\dot{\mathbf{Q}}=-2 \mathbf{Q} \text {. }
$$

It then follows that $\lim _{t \rightarrow \infty}\left(\mathbf{A}_{2}(t)-\mathbf{A}_{1}(t)\right)=\overline{\mathbf{D}}$ exponentially, where $\overline{\mathbf{D}}=\frac{1}{2}(I+R(-\phi)) \mathbf{D}$. Straightforward calculation shows that $\overline{\mathbf{D}}=\left[\begin{array}{cc}\frac{1+\cos \phi}{2} & \frac{\sin \phi}{2} \\ -\frac{\sin \phi}{2} & \frac{1+\cos \phi}{2}\end{array}\right] \mathbf{D}=$ $\cos \frac{\phi}{2} R(-\phi / 2) \mathbf{D}$. This verifies (I).

(II) Since $\lim _{t \rightarrow \infty}\left(\mathbf{A}_{2}(t)-\mathbf{A}_{1}(t)-\overline{\mathbf{D}}\right)=0$ exponentially fast, it follow from (11) that $\lim _{t \rightarrow \infty}\left(\dot{\mathbf{A}}_{2}(t)-\dot{\mathbf{A}}_{1}(t)\right)=0$ exponentially fast. Therefore, (II) is proven.

(III) Note that the desired formation is determined by $\mathbf{D}$, but the actual final formation is determined by $\overline{\mathbf{D}} \neq \mathbf{D}$. Therefore, for the case of $\mathbf{D} \neq 0$, the final formation is distorted from the desired one. We define the distance error between the actual final relative position and the desired one as $\delta \mathbf{D}=|\|\overline{\mathbf{D}}\|-\|\mathbf{D}\||$. It is not hard to show that $\|\overline{\mathbf{D}}\|=$ $\cos \frac{\phi}{2}\|\mathbf{D}\|$. Therefore, for small $|\phi|, \delta \mathbf{D}=\left(1-\cos \frac{\phi}{2}\right)\|\mathbf{D}\|=$ $2 \sin ^{2}(\phi / 4)\|\mathbf{D}\| \approx \frac{\phi^{2}}{8}\|\mathbf{D}\|$. This shows that $\delta \mathbf{D}=O\left(\phi^{2}\right)$, as $\phi \rightarrow 0$. In addition, by noting that $\overline{\mathbf{D}}=\cos \frac{\phi}{2} R(-\phi / 2) \mathbf{D}$, we know that the angular error between the actual final relative position and the desired one is $\frac{|\phi|}{2}$.

On the other hand, if $\mathbf{D}=0$, it follows from the definition of $\overline{\mathbf{D}}$ that rendezvous is still achieved, i.e., $\lim _{t \rightarrow \infty}\left(\mathbf{A}_{2}(t)-\right.$ $\left.\mathbf{A}_{1}(t)\right)=0$.

(IV) It follows from (2) that $\lim _{t \rightarrow \infty} \dot{\mathbf{A}}_{1}(t)=$ $\lim _{t \rightarrow \infty} \dot{\mathbf{A}}_{2}(t)=\frac{1}{2}(R(-\phi)-I) \mathbf{D}=$ $\left[\begin{array}{cc}\frac{\cos \phi-1}{2} & \frac{\sin \phi}{2} \\ -\frac{\sin \phi}{2} & \frac{\cos \phi-1}{2}\end{array}\right] \mathbf{D}=\sin \frac{\phi}{2}\left[\begin{array}{cc}\sin \frac{\phi}{2} & \cos \frac{\phi}{2} \\ -\cos \frac{\phi}{2} & \sin \frac{\phi}{2}\end{array}\right] \mathbf{D}$. This further implies when $\mathbf{D} \neq 0$ that $\lim _{t \rightarrow \infty}\left\|\dot{\mathbf{A}}_{1}(t)\right\|=$ $\lim _{t \rightarrow \infty}\left\|\dot{\mathbf{A}}_{2}(t)\right\|=O(|\phi|)$, as $\phi \rightarrow 0$. Therefore, for the case of $\mathbf{D} \neq 0$, the absolute velocities of both agents converge to the same nonzero constant exponentially fast. For the case of $\mathbf{D}=0$, the absolute velocities of both agents converge to zero exponentially fast.

(V) For the case of $\mathbf{D} \neq 0$, the conclusions are obvious due to (IV). If $\mathbf{D}=0$, the problem reduces to a standard average consensus problem [9].

We next describe simulations which illustrate the validity of Theorem 1. We first consider the case of $\mathbf{D} \neq 0$. In particular, $\mathbf{D}=[0,-5]^{\mathrm{T}}, \phi=-0.2 \pi$, and the two agents start from the desired formation. Figure 2 shows the positions of the agents during the time interval $[0,10]$. Due to the existence of mismatched directions, the agents keep moving with non-zero constant absolute velocities and the

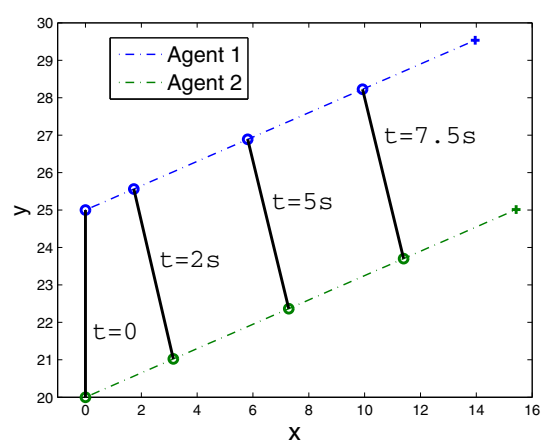

(a) The dotted lines denote the trajectories of the positions of the agents. The circles and the solid black lines denote respectively, the positions of the agents and the formation shape at $\mathrm{t}=0 \mathrm{~s}, \mathrm{t}=2 \mathrm{~s}, \mathrm{t}=5 \mathrm{~s}$, and $\mathrm{t}=7.5$ s.

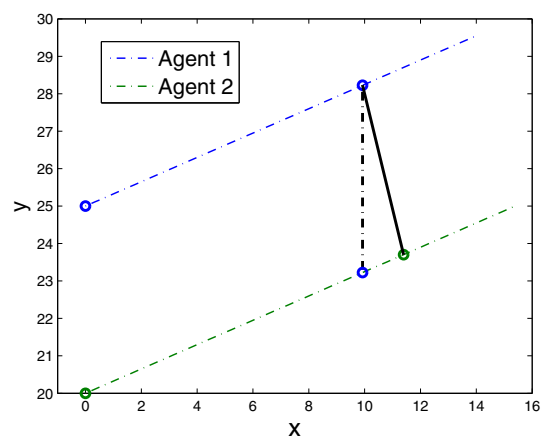

(b) The solid black line and the dotted black line denote respectively, the actual formation shape and desired formation shape at $\mathrm{t}=7.5 \mathrm{~s}$.

Fig. 2. Two-dimensional case with $\mathbf{D} \neq 0$.

final formation is distorted from the desired one. This is in contrast to the case with matched directions where the agents converge to the desired stationary formation.

\section{B. Estimation and compensation algorithms}

In this section, we first consider the estimation of $\phi$. We shall show later how the use of an estimate of $\phi$ can eliminate the problem caused by the directional mismatch.

Since each agent in the steady state will perceive a discrepancy in its own coordinate basis between the desired formation and the actual formation, with an angular error of $\frac{\phi}{2}$, each agent is able to estimate $\phi$ separately using its own available information. (Of course, since the estimation is to be used to eliminate the mismatch problem, it is desirable to do this before steady state is reached, and the approach for this is set out in more detail below). Without loss of generality, we let agent 2 be responsible for the estimation of $\phi$. The following compensation control algorithm is proposed

$$
\begin{gathered}
{ }^{1} \dot{\mathbf{A}}_{1}=\mathbf{A}_{2}-\mathbf{A}_{1}-\mathbf{D}, \\
{ }^{2} \dot{\mathbf{A}}_{2}=R(\phi)\left(\mathbf{A}_{1}-\mathbf{A}_{2}\right)+\mathbf{D}+\mathbf{U},
\end{gathered}
$$


where $\mathbf{U} \in \mathbb{R}^{2}$ denotes a compensation input. We next specify how to design the estimation of $\phi$ and use the estimated information on $\phi$ to compensate the original mismatched algorithm such that the desired formation is achieved.

We assume that the relative position information of agent 2 is available for agent 2 and expressed in the coordinate basis of agent 2 , i.e., ${ }^{2} \mathbf{A}_{2}-{ }^{2} \mathbf{A}_{1}=\left[{ }^{2} x_{2}-{ }^{2} x_{1},{ }^{2} y_{2}-{ }^{2} y_{1}\right]^{\mathrm{T}}$ is available for agent 2 .

It follows from (5a) and the fact ${ }^{2} \dot{\mathbf{A}}_{1}=R(\phi){ }^{1} \dot{\mathbf{A}}_{1}$ that

$$
{ }^{2} \dot{\mathbf{A}}_{1}=R(\phi)\left(\mathbf{A}_{2}-\mathbf{A}_{1}\right)-R(\phi) \mathbf{D} .
$$

Therefore, using $(5 b)$, we know that

$$
{ }^{2} \dot{\mathbf{A}}_{2}-{ }^{2} \dot{\mathbf{A}}_{1}=-2\left({ }^{2} \mathbf{A}_{2}-{ }^{2} \mathbf{A}_{1}\right)+R(\phi) \mathbf{D}+\mathbf{D}+\mathbf{U} .
$$

It then follows that for any estimate $\hat{\phi}(t)$,

$$
\begin{aligned}
R(\phi) \mathbf{D}-R(\hat{\phi}(t)) \mathbf{D} & ={ }^{2} \dot{\mathbf{A}}_{2}-{ }^{2} \dot{\mathbf{A}}_{1}+2\left({ }^{2} \mathbf{A}_{2}-{ }^{2} \mathbf{A}_{1}\right) \\
& -R(\hat{\phi}(t)) \mathbf{D}-\mathbf{D}-\mathbf{U} .
\end{aligned}
$$

In the light of this equation, we propose the following estimation algorithm for $\hat{\phi}(t)$ :

$$
\begin{aligned}
\dot{z}= & k\left(2 d_{x}\left({ }^{2} y_{2}-{ }^{2} y_{1}\right)-2 d_{y}\left({ }^{2} x_{2}-{ }^{2} x_{1}\right)\right. \\
& \left.-\left(d_{x}^{2}+d_{y}^{2}\right) \sin \hat{\phi}-\left(d_{x} u_{y}-d_{y} u_{x}\right)\right), \\
& \hat{\phi}=z+k\left(d_{x}\left({ }^{2} y_{2}-{ }^{2} y_{1}\right)-d_{y}\left({ }^{2} x_{2}-{ }^{2} x_{1}\right)\right),
\end{aligned}
$$

where $k$ is a positive constant and $\mathbf{U}=\left[u_{x}, u_{y}\right]^{\mathrm{T}}$.

We next present the following result regarding the convergence of $\hat{\phi}(t)$ using the estimator (7) for the compensation algorithm (5).

Theorem 2: Consider the estimator (7) for the compensation algorithm (5). Suppose that $|\phi|<\frac{\pi}{4}$ and $|\hat{\phi}(0)| \leq \frac{\pi}{4}$. It follows that $\lim _{t \rightarrow \infty} \hat{\phi}(t)=\phi$.

Proof: Denote $\widetilde{\mathbf{D}}=\left[\begin{array}{cc}0 & -1 \\ 1 & 0\end{array}\right]$ D. It follows by an easy direct calculation that

$$
\widetilde{\mathbf{D}}^{\mathrm{T}}(R(\phi)-R(\hat{\phi}(t))) \mathbf{D}=-(\sin \hat{\phi}-\sin \phi)\|\mathbf{D}\|^{2} .
$$

Also note that (7) can be written as

$$
\begin{aligned}
\dot{z} & =k \widetilde{\mathbf{D}}^{\mathrm{T}}\left(2\left({ }^{2} \mathbf{A}_{2}-{ }^{2} \mathbf{A}_{1}\right)-R(\hat{\phi}(t)) \mathbf{D}-\mathbf{D}-\mathbf{U}\right), \\
\hat{\phi}(t) & =z(t)+k \widetilde{\mathbf{D}}^{\mathrm{T}}\left({ }^{2} \mathbf{A}_{2}-{ }^{2} \mathbf{A}_{1}\right) .
\end{aligned}
$$

It then follows that

$$
\begin{aligned}
\dot{\hat{\phi}}= & k \widetilde{\mathbf{D}}^{\mathrm{T}}\left({ }^{2} \dot{\mathbf{A}}_{2}-{ }^{2} \dot{\mathbf{A}}_{1}+2\left({ }^{2} \mathbf{A}_{2}-{ }^{2} \mathbf{A}_{1}\right)\right. \\
& -R(\hat{\phi}(t)) \mathbf{D}-\mathbf{D}-\mathbf{U}) \\
= & k \widetilde{\mathbf{D}}^{\mathrm{T}}(R(\phi)-R(\hat{\phi}(t))) \mathbf{D} \\
= & -k(\sin \hat{\phi}-\sin \phi)\|\mathbf{D}\|^{2} \\
= & -2 k \sin \left(\frac{\hat{\phi}-\phi}{2}\right) \cos \left(\frac{\hat{\phi}+\phi}{2}\right)\|\mathbf{D}\|^{2} .
\end{aligned}
$$

Choose a Lyapunov function candidate as $V=(\hat{\phi}-$ $\phi)^{2}$. Since $|\hat{\phi}(0)| \leq \frac{\pi}{4}$ and $|\phi|<\frac{\pi}{4}$, it follows that $\cos \left(\frac{\hat{\phi}(0)+\phi}{2}\right)>0$. This implies that $\dot{V} \leq 0$ at $t=0$. We thus know that $\left|\hat{\phi}\left(0^{+}\right)-\phi\right| \leq|\hat{\phi}(0)-\phi|<\frac{\pi}{2}$. Noting that $|\hat{\phi}(t)+\phi|=|\hat{\phi}(t)-\phi+2 \phi|<|\hat{\phi}(t)-\phi|+\frac{\pi}{2}$, it follows that $\left|\hat{\phi}\left(0^{+}\right)+\phi\right|<\pi$ and $\cos \left(\frac{\hat{\phi}\left(0^{+}\right)+\phi}{2}\right)>0$. Therefore, $\dot{V} \leq 0$ at $t=0^{+}$. We next use this observation to show that $|\hat{\phi}(t)-\phi|<\frac{\pi}{2}$, for all $t \geq 0$ using a contradiction argument. Suppose it is not true. Then, there must exist a finite time instant $T$ for which $|\hat{\phi}(T)-\phi|=\frac{\pi}{2}$ and $|\hat{\phi}(t)-\phi|<\frac{\pi}{2}$ for all $t \in[0, T)$. Based on the continuity, there must exist a neighborhood $[T-\delta, T)$ such that $|\hat{\phi}(t)-\phi|$ is increasing during $t \in[T-\delta, T)$, where $\delta>0$. However, based on the definition of $T$ and the observation for the case of $t=0^{+}$, we know that $\dot{V}(t) \leq 0$, for all $t \in[0, T)$. This shows that $|\hat{\phi}(t)-\phi|$ is non-increasing during $t \in[0, T)$ and therefore indicates a contradiction. Therefore, we know that $\dot{V} \leq 0$, for all $t \geq 0$. Then, based on the Lasalle invariance principle, we can conclude that $\lim _{t \rightarrow \infty} \hat{\phi}(t)=\phi$.

Next, we show how to use the estimated information on $\phi$ to compensate the original mismatched algorithm such that the desired formation is achieved. Specifically, the following compensation control algorithm is proposed

$$
\mathbf{U}(t)=-\mathbf{D}+R(\hat{\phi}(t)) \mathbf{D}
$$

where $\hat{\phi}(t)$ is obtained by the estimator (7).

We next show that $\mathbf{A}_{2}(t)-\mathbf{A}_{1}(t)$ converges to the desired formation using compensation input (8) and the estimator (7).

Theorem 3: Consider the algorithm (5) with compensation input (8) and the estimator (7). Suppose that $|\phi|<\frac{\pi}{4}$ and $|\hat{\phi}(0)| \leq \frac{\pi}{4}$. Then $\lim _{t \rightarrow \infty} \hat{\phi}(t)=\phi, \lim _{t \rightarrow \infty}\left(\mathbf{A}_{2}(t)-\right.$ $\left.\mathbf{A}_{1}(t)\right)=\mathbf{D}$, and $\lim _{t \rightarrow \infty} \mathbf{V}_{1}(t)=\lim _{t \rightarrow \infty} \mathbf{V}_{2}(t)=0$.

Proof: It is not hard to show from (5) and (8) that

$$
\dot{\mathbf{A}}_{1}-\dot{\mathbf{A}}_{2}=-2\left(\mathbf{A}_{1}-\mathbf{A}_{2}+\mathbf{D}\right)+\mathbf{D}-R(\hat{\phi}-\phi) \mathbf{D} .
$$

Noting that we have shown from Theorem 2 that $\lim _{t \rightarrow \infty} R(\hat{\phi}(t)-\phi)=I$, it then follows from the inputto-state stability property ( [16]) that $\lim _{t \rightarrow \infty}\left(\mathbf{A}_{2}(t)-\right.$ $\left.\mathbf{A}_{1}(t)\right)=\mathbf{D}$. It is then trivial to show that $\lim _{t \rightarrow \infty} \mathbf{V}_{1}(t)=$ $\lim _{t \rightarrow \infty} \mathbf{V}_{2}(t)=0$.

\section{FORMATIONS IN THREE-DIMENSIONAL SPACE}

To this point, we have considered undirected formations in a two-dimensional plane. We now show that similar results hold for the two agent case in a three-dimensional ambient space. In this section, we abuse the notation as $\mathbf{A}_{1}=\left[x_{1}, y_{1}, z_{1}\right]^{\mathrm{T}} \in \mathbb{R}^{3}, \mathbf{A}_{2}=\left[x_{2}, y_{2}, z_{2}\right]^{\mathrm{T}} \in \mathbb{R}^{3}$, and $\mathbf{D}=\left[d_{x}, d_{y}, d_{z}\right]^{\mathrm{T}} \in \mathbb{R}^{3}$. We still assume that the global coordinates coincide with the coordinate basis of agent 1 and suppose that the rotation from the coordinate of agent 1 to that of agent 1 is represented by an Euler axis and angle [17]. An illustration is given in Figure 3.

Then, in each agent's own coordinate basis, the actual kinematics of each agent with mismatched directions are given by

$$
{ }^{1} \dot{\mathbf{A}}_{1}=\mathbf{A}_{2}-\mathbf{A}_{1}-\mathbf{D}
$$




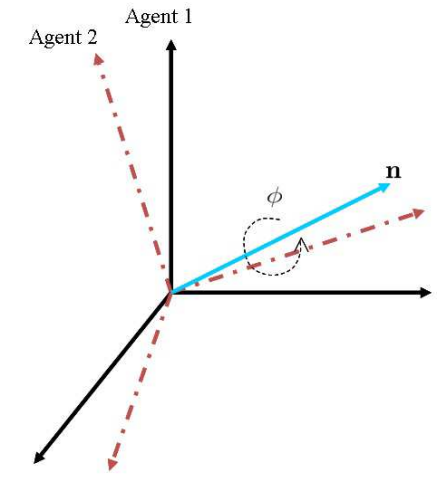

Fig. 3. Coordinates of agents 1 and 2 .

$$
{ }^{2} \dot{\mathbf{A}}_{2}=R(\mathbf{n}, \phi)\left(\mathbf{A}_{1}-\mathbf{A}_{2}\right)+\mathbf{D}
$$

where $R(\mathbf{n}, \phi)$ is the rotation matrix [18], $\mathbf{n}=\left[\begin{array}{l}n_{1} \\ n_{2} \\ n_{3}\end{array}\right] \in \mathbb{R}^{3}$ is a unit vector and $\phi \in(-\pi, \pi]$ is an angle. In particular, $R(\mathbf{n}, \phi)=I+\sin \phi[\mathbf{n}]_{\times}+(1-\cos \phi)\left([\mathbf{n}]_{\times}\right)^{2}$, where $[\mathbf{n}]_{\times}=$ $\left[\begin{array}{ccc}0 & -n_{3} & n_{2} \\ n_{3} & 0 & -n_{1} \\ -n_{2} & n_{1} & 0\end{array}\right]$ denotes the cross product matrix of $\mathbf{n}$. It is not hard to show that (9) can be written as

$$
\begin{gathered}
\dot{\mathbf{A}}_{1}=\mathbf{A}_{2}-\mathbf{A}_{1}-\mathbf{D}, \\
\dot{\mathbf{A}}_{2}=\mathbf{A}_{1}-\mathbf{A}_{2}+R(\mathbf{n},-\phi) \mathbf{D},
\end{gathered}
$$

We next present the following result on the case of threedimensional ambient space.

Theorem 4: Consider the mismatched formation shape control algorithm (9). Suppose $\mathbf{n} \neq 0$ and $\phi \neq 0$ and constants. It follows that

[Intra-formation motion]

(I) The agents converge to a fixed formation exponentially fast. In particular, $\lim _{t \rightarrow \infty}\left(\mathbf{A}_{2}(t)-\mathbf{A}_{1}(t)\right)=\frac{1}{2}(I+$ $R(\mathbf{n},-\phi)) \mathbf{D}$.

(II) The relative velocities of the agents converge to zero exponentially fast, i.e., $\lim _{t \rightarrow \infty}\left(\mathbf{V}_{2}(t)-\mathbf{V}_{1}(t)\right)=0$.

(III) If $\mathbf{D} \neq 0$, the final formation is distorted from the desired one. The distance error between the actual final relative position and the desired one is $O\left(\phi^{2}\right)$, as $\phi \rightarrow$ 0 . If $\mathbf{D}=0$, then rendezvous is still achieved, i.e., $\lim _{t \rightarrow \infty}\left(\mathbf{A}_{2}(t)-\mathbf{A}_{1}(t)\right)=0$.

[Whole-formation motion]

(IV) If $\mathbf{D} \neq 0$, the absolute velocities of both agents converge to the same nonzero constant exponentially fast. In particular, $\lim _{t \rightarrow \infty}\left\|\mathbf{V}_{1}(t)\right\|=\lim _{t \rightarrow \infty}\left\|\mathbf{V}_{2}(t)\right\|=$ $O(|\phi|)$, as $\phi \rightarrow 0$. If $\mathbf{D}=0$, the absolute velocities of both agents converge to zero exponentially fast.

(V) If $\mathbf{D} \neq 0$, the agent positions $\mathbf{A}_{1}(t)$ and $\mathbf{A}_{2}(t)$ are neither convergent nor bounded. If $\mathbf{D}=0$, $\lim _{t \rightarrow \infty} \mathbf{A}_{1}(t)=\lim _{t \rightarrow \infty} \mathbf{A}_{2}(t)=\frac{\mathbf{A}_{1}(0)+\mathbf{A}_{2}(0)}{2}$, where $\mathbf{A}_{1}(0)$ and $\mathbf{A}_{2}(0)$ are the initial states of agents 1 and 2 .
Proof:

(I) Define $\mathbf{Q}=\mathbf{A}_{1}-\mathbf{A}_{2}+\frac{1}{2}(I+R(\mathbf{n},-\phi)) \mathbf{D}$. It follows from (3) that

$$
\dot{\mathbf{Q}}=-2 \mathbf{Q}
$$

It then follows that $\lim _{t \rightarrow \infty}\left(\mathbf{A}_{2}(t)-\mathbf{A}_{1}(t)\right)=\overline{\mathbf{D}}$ exponentially, where $\overline{\mathbf{D}}=\frac{1}{2}(I+R(\mathbf{n},-\phi)) \mathbf{D}$. This verifies (I).

(II) Since $\lim _{t \rightarrow \infty}\left(\mathbf{A}_{2}(t)-\mathbf{A}_{1}(t)-\overline{\mathbf{D}}\right)=0$ exponentially fast, it follow from (11) that $\lim _{t \rightarrow \infty}\left(\dot{\mathbf{A}}_{2}(t)-\dot{\mathbf{A}}_{1}(t)\right)=0$ exponentially fast. Therefore, (II) is proven.

(III) Note that the desired formation is determined by $\mathbf{D}$, but the actual final formation is determined by $\overline{\mathbf{D}} \neq \mathbf{D}$. Same as the case of a two-dimensional plane, we define the distance error between the actual final relative position and the desired one as $\delta \mathbf{D}=|\|\overline{\mathbf{D}}\|-\|\mathbf{D}\||$. Note that $R(\mathbf{n}, \phi)=$ $\left[\begin{array}{ccc}c+n_{1}^{2}(1-c) & n_{1} n_{2}(1-c)-n_{3} s & n_{1} n_{3}(1-c)+n_{2} s \\ n_{1} n_{2}(1-c)+n_{3} s & c+n_{2}^{2}(1-c) & n_{2} n_{3}(1-c)-n_{1} s \\ n_{1} n_{3}(1-c)-n_{2} s & n_{2} n_{3}(1-c)+n_{1} s & c+n_{3}^{2}(1-c)\end{array}\right]$, where $c=\cos \phi$ and $s=\sin \phi$. Denote $\bar{c}=\cos (\phi / 2)$ and $\bar{s}=\sin (\phi / 2)$. It follows that

$$
\begin{aligned}
& I+R(\mathbf{n},-\phi) \\
& =2\left[\begin{array}{ccc}
\bar{c}^{2}+\bar{s}^{2} n_{1}^{2} & n_{1} n_{2} \bar{s}^{2}+n_{3} \bar{s} \bar{c} & n_{1} n_{3} \bar{s}^{2}-n_{2} \bar{s} \bar{c} \\
n_{1} n_{2} \bar{s}^{2}-n_{3} \bar{s} \bar{c} & \bar{c}^{2}+\bar{s}^{2} n_{2}^{2} & n_{2} n_{3} \bar{s}^{2}+n_{1} \bar{s} \bar{c} \\
n_{1} n_{3} \bar{s}^{2}+n_{2} \bar{s} \bar{c} & n_{2} n_{3} \bar{s}^{2}-n_{1} \bar{s} \bar{c} & \bar{c}^{2}+\bar{s}^{2} n_{3}^{2}
\end{array}\right] \\
& =2 \bar{s}^{2}\left[\begin{array}{ccc}
n_{1}^{2} & n_{1} n_{2} & n_{1} n_{3} \\
n_{1} n_{2} & n_{2}^{2} & n_{2} n_{3} \\
n_{1} n_{3} & n_{2} n_{3} & n_{3}^{2}
\end{array}\right]+2 \bar{c}\left[\begin{array}{ccc}
\bar{c} & n_{3} \bar{s} & -n_{2} \bar{s} \\
-n_{3} \bar{s} & \bar{c} & n_{1} \bar{s} \\
n_{2} \bar{s} & -n_{1} \bar{s} & \bar{c}
\end{array}\right] \\
& =2(1-\bar{c})\left[\begin{array}{ccc}
n_{1}^{2} & n_{1} n_{2} & n_{1} n_{3} \\
n_{1} n_{2} & n_{2}^{2} & n_{2} n_{3} \\
n_{1} n_{3} & n_{2} n_{3} & n_{3}^{2}
\end{array}\right]+2 \bar{c} \\
& \times\left(\left[\begin{array}{ccc}
\bar{c} & n_{3} \bar{s} & -n_{2} \bar{s} \\
-n_{3} \bar{s} & \bar{c} & n_{1} \bar{s} \\
n_{2} \bar{s} & -n_{1} \bar{s} & \bar{c}
\end{array}\right]+(1-\bar{c})\left[\begin{array}{ccc}
n_{1}^{2} & n_{1} n_{2} & n_{1} n_{3} \\
n_{1} n_{2} & n_{2}^{2} & n_{2} n_{3} \\
n_{1} n_{3} & n_{2} n_{3} & n_{3}^{2}
\end{array}\right]\right) \\
& =4 \sin ^{2}(\phi / 2)\left[\begin{array}{ccc}
n_{1}^{2} & n_{1} n_{2} & n_{1} n_{3} \\
n_{1} n_{2} & n_{2}^{2} & n_{2} n_{3} \\
n_{1} n_{3} & n_{2} n_{3} & n_{3}^{2}
\end{array}\right]+2 \cos (\phi / 2) R(\mathbf{n},-\phi / 2) .
\end{aligned}
$$

Therefore, for small $|\phi|$, it is not hard to show that $\delta \mathbf{D} \approx$ $\left(1-\cos \frac{\phi}{2}\right)\|\mathbf{D}\|=2 \sin ^{2}(\phi / 4)\|\mathbf{D}\| \approx \frac{\phi^{2}}{8}\|\mathbf{D}\|$. This shows that $\delta \mathbf{D}=O\left(\phi^{2}\right)$, as $\phi \rightarrow 0$.

On the other hand, if $\mathbf{D}=0$, it follows from the definition of $\overline{\mathbf{D}}$ that rendezvous is still achieved, i.e., $\lim _{t \rightarrow \infty}\left(\mathbf{A}_{2}(t)-\right.$ $\left.\mathbf{A}_{1}(t)\right)=0$.

(IV) It follows from (2) that $\lim _{t \rightarrow \infty} \dot{\mathbf{A}}_{1}(t)=$ $\lim _{t \rightarrow \infty} \dot{\mathbf{A}}_{2}(t)=\frac{1}{2}(R(\mathbf{n},-\phi)-I) \mathbf{D}=\sin \frac{\phi}{2}\left(-\cos \frac{\phi}{2}[\mathbf{n}]_{\times}+\right.$ $\left.\sin \frac{\phi}{2}\left([\mathbf{n}]_{\times}\right)^{2}\right) \mathbf{D}$. This further implies when $\mathbf{D} \neq 0$ that $\lim _{t \rightarrow \infty}\left\|\dot{\mathbf{A}}_{1}(t)\right\|=\lim _{t \rightarrow \infty}\left\|\dot{\mathbf{A}}_{2}(t)\right\|=O(|\phi|)$, as $\phi \rightarrow 0$. Therefore, for the case of $\mathbf{D} \neq 0$, the absolute velocities of both agents converge to the same nonzero constant exponentially fast. For the case of $\mathbf{D}=0$, the absolute velocities of both agents converge to zero exponentially fast.

(V) For the case of $\mathbf{D} \neq 0$, the conclusions are obvious due to (IV). If $\mathbf{D}=0$, the problem reduces to a standard average consensus problem [9]. 


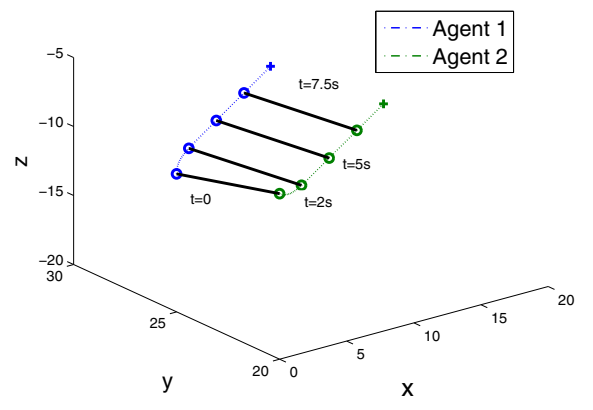

(a) The circles and the solid black lines denote respectively, the positions of the agents and the formation shape at $\mathrm{t}=0 \mathrm{~s}, \mathrm{t}=2 \mathrm{~s}, \mathrm{t}=5 \mathrm{~s}$, and $\mathrm{t}=7.5 \mathrm{~s}$.

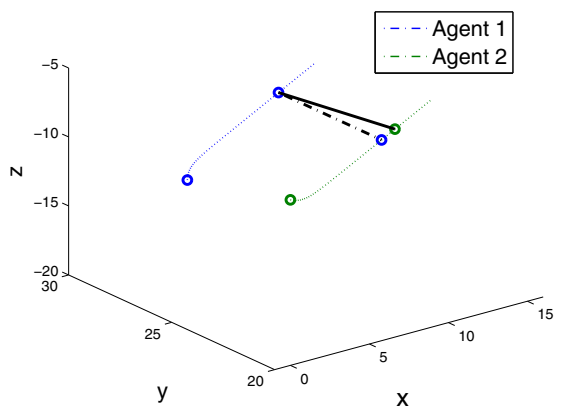

(b) The solid black line and the dotted black line denote respectively, the actual formation shape and desired formation shape at $\mathrm{t}=7.5 \mathrm{~s}$.

Fig. 4. Three-dimensional case with $\mathbf{D} \neq 0$.

We next describe simulations which illustrate the validity of Theorem 4. We first consider the case of $\mathbf{D} \neq 0$. In particular, $\mathbf{D}=[0,-5,0]^{\mathrm{T}}, \phi=-0.2 \pi$, and the two agents start from the desired formation. Figure 4 shows the positions of the agents during the time interval $[0,10]$. Due to the existence of mismatched directions, the agents keep moving with non-zero constant absolute velocities and the final formation is distorted from the desired one. This is similar to the case of two-dimensional plane.

\section{CONCLuding Remarks}

This paper studied the formation shape control problem with mismatched directions. Such a mismatch is a consequence of the fact that it is not physically realistic to claim that all agents have common error-free knowledge of where north is. We examined the consequences of the mismatched directions on a standard formation shape control algorithm. The two agent case in a two-dimensional plane was first studied and we showed that the agents converge to a fixed, but distorted formation exponentially fast. Unlike the matched case, the formation is not asymptotically stationary. The shape error between the actual final formation and the desired formation was explicitly established. We then proposed estimation and compensation algorithms such that the desired formation shape is achieved exponentially fast.
The exponential property provides a measure of robustness to noise. Although not covered in the paper, the ideas are easily extended to more complicated formations. The extensions to the case of a three-dimensional space were indicated also. Simulations are provided to validate the theoretical results in the absence of noise. The results of this paper raise a number of open issues, including considering other agent models, such as second order agents or unicycle agents, considering the effect of bias in distances simultaneously with mismatch in the direction of north, and studying estimation and compensation algorithms for the three-dimensional ambient space case.

\section{REFERENCES}

[1] D. V. Dimarogonas and K. J. Kyriakopoulos, "On the rendezvous problem for multiple nonholonomic agents," IEEE Transactions on Automatic Control, vol. 52, no. 5, pp. 916-922, 2007.

[2] D. Sieber, F. Deroo, and S. Hirche, "Formation-based approach for multi-robot cooperative manipulation based on optimal control design," in IEEE/RSJ International Conference on Intelligent Robots and Systems (IROS), Tokyo, Japan, 2013, pp. 5227-5233.

[3] W. Ren, "Multi-vehicle consensus with a time-varying reference state," Systems \& Control Letters, vol. 56, no. 7, pp. 474-483, 2007.

[4] G. Shi and Y. Hong, "Global target aggregation and state agreement of nonlinear multi-agent systems with switching topologies," Automatica, vol. 45 , no. 5, pp. $1165-1175,2009$.

[5] Y. Hatano and M. Mesbahi, "Agreement over random networks," IEEE Transations on Automatic Control, vol. 50, no. 11, pp. 1867-1872, 2005.

[6] L. Moreau, "Stability of multi-agent systems with time-dependent communication links," IEEE Transactions on Automatic Control, vol. 50, no. 2, pp. 169-182, 2005.

[7] Z. Lin, M. Broucke, and B. Francis, "Local control strategies for groups of mobile autonomous agents," IEEE Transactions on Automatic Control, vol. 49, no. 4, pp. 622-629, 2004.

[8] B. Jiang, M. Deghat, and B. D. O. Anderson, "Translational velocity consensus using distance-only measurements," in Proceedings of 2013th IEEE Conference on Decision and Control, Florence, Italy, 2013, pp. 2746-2751.

[9] R. Olfati-Saber, J. A. Fax, and R. M. Murray, "Consensus and cooperation in networked multi-agent systems," Proceedings of the IEEE, vol. 95, no. 1, pp. 215-233, 2007.

[10] W. Ren, R. W. Beard, and E. M. Atkins, "Information consensus in multivehicle cooperative control: collective group behavior through local interaction," IEEE Control Systems Magazine, vol. 27, no. 2, pp. 71-82, 2007.

[11] L. Krick, M. E. Broucke, and B. A. Francis, "Stabilization of infinitesimally rigid formations of multi-robot networks," International Journal of Control, vol. 82, no. 3, pp. 49-95, 2009.

[12] M. Cao, A. S. Morse, C. Yu, B. D. O. Anderson, and S. Dasgupta, "Maintaining a directed, triangular formation of mobile autonomous agents," Communications in Information and Systems, vol. 11, no. 1, pp. 1-16, 2011.

[13] K.-K. Oh and H.-S. Ahn, "Formation control and network localization via orientation alignment," IEEE Transactions on Automatic Control, vol. 59, no. 2, pp. 540-545, 2014.

[14] M. A. Belabbas, S. Mou, A. S. Morse, and B. D. O. Anderson, "Robustness issues with undirected formations," in Proceedings of the 2012th IEEE Conference on Decision and Control, Maui, Hawaii, USA, 2012, pp. 1445-1450.

[15] Z. Sun, S. Mou, B. D. O. Anderson, and A. S. Morse, "Non robustness of gradient control for 3-d undirected formations with distance mismatch," in Proceedings of 2013th Australian Control Conference, Perth, Australia, 2013, pp. 369-374.

[16] H. K. Khalil, Nonlinear Systems. Prentice Hall, 2002.

[17] M. D. Shuster, "A survey of attitude representations," The Journal of the Astronautical Sciences, vol. 41, no. 4, pp. 439-517, 1993.

[18] N. Chaturvedi, A. Sanyal, and N. McClamroch, "Rigid-body attitude controls," IEEE Control Systems Magazine, vol. 31, no. 3, pp. 30-51, 2011. 